Willan Simões Clagnan'

JURANDYR MOREIRA DE ANDRADE ${ }^{2}$

Hélo Humberto Angotti Carrara ${ }^{3}$

Daniel Guimarães Tiezzi'

Francisco José CANDIDO dos Reis ${ }^{4}$

Heitor Ricardo Cosiski Marana ${ }^{1}$

Renato Antonio Abrão ${ }^{5}$

\title{
Idade como fator independente de prognóstico no câncer de mama
}

\author{
Age as an independent prognostic factor in breast cancer
}

Artigos originais

Palavras-chave

Fatores etários

Fatores de risco

Prognóstico

Neoplasias mamárias/epidemiologia

Keywords

Age factors

Risk factors

Prognosis

Breast neoplasms/epidemiology

\section{Resumo}

OBJETIVO: comparar as características epidemiológicas e clínicas e a evolução pós-tratamento de mulheres com câncer de mama diagnosticadas antes ou após os 40 anos de idade. MÉTODOS: foi realizado um estudo retrospectivo, tipo caso-controle, com análise de informações obtidas dos prontuários de pacientes atendidas entre janeiro de 1994 e junho de 2004. Excluímos os casos com carcinomas intraductais e no estádio IV. Foram formados três grupos: pacientes com menos de 40 anos no diagnóstico (n=72), pacientes entre 40 e 50 anos (n=68) e pacientes com mais de 50 anos ( $n=75)$. Foram coletadas e analisadas informações sobre a idade no momento do diagnóstico, maior diâmetro das lesões, estadiamento clínico, tipo, grau histológico, presença de receptores hormonais e o estado dos linfonodos. $\bigcirc$ teste do $x^{2}$ foi empregado para variáveis qualitativas. Para as variáveis quantitativas que não apresentam distribuição normal (como número de gânglios axilares acometidos por metástases e tempo de seguimento), foi utilizado o teste de Kruskal-Wallis. Para a construção das curvas de sobrevida livre de doença e sobrevida global, foi utilizado o teste log-rank. RESULTADOS: não houve diferença na distribuição por estádios, no grau de diferenciação tumoral ou na distribuição dos tipos histológicos, na expressão de receptores de estrogênio ou c-erb-B2 entre os grupos. Encontramos diferença na expressão de receptores de estrogênio, que foi menos freqüente no grupo com menos de 40 anos em relação ao grupo com mais de 50 anos $(36,2$ versus $58,4 \%)$. Não houve diferença nos diâmetros tumorais, que foram em média de 5, 1, 4,7 e $5 \mathrm{~cm}$. Não encontramos diferença nas taxas de acometimento de linfonodos axilares, que foram de 63,9,46,9 e 50\%, respectivamente nas pacientes com menos de 40 anos, pacientes entre 40 e 50 anos e pacientes com mais de 50 anos. A média de seguimento foi de 54 meses nos três grupos. Houve recidiva da doença em 22,6\% das pacientes com menos de 40 anos, $60 \%$ das pacientes entre 40 e 50 anos e 22,6\% das pacientes com mais de 50 anos, com diferença significante $(p<0,0001)$. Encontramos taxa de óbito pela doença de 46,9\% nas pacientes com menos de 40 anos, mais elevada que nas pacientes entre 40 e 50 anos $(26,9 \%)$ e pacientes com mais de 50 anos $(22,6 \%), p=0,0019$. A análise logística mostrou que somente a idade inferior a 40 anos e a presença de mais de um gânglio axilar acometido por metástase foram fatores independentes para risco de morte pela doença. CONCLUSÕES: a idade menor que 40 anos é um fator de risco independente no câncer de mama. Os indicadores de prognóstico tradicionais como estádio, diâmetro tumoral, comprometimento axilar e receptores hormonais não mostraram associação com a evolução das pacientes.

\section{Abstract}

PURPOSE: to compare the epidemiologic and clinical characteristics, and the follow-up of breast cancer in women diagnosed under and over 40 years of age. METHODS: a retrospective study, case-control type, with analysis of information obtained from medical records of patients attended from January 1994 to June 2004. Cases of intraductal carcinoma and at stage IV were excluded. Three groups were formed: patients under 40 years old at the diagnosis $(n=72)$; patients between 40 and $50(n=68)$ and patients over $50(n=75)$. Data about age at the moment of diagnosis, lesion largest diameter, clinical stage, type, histological grade, presence of hormonal receptors and state of the lymph nodes were collected and analyzed. The $x^{2}$ test was used for qualitative variables. For quantitative variables without
Willian Simões Clagnan Hospital das Clínicas da Faculdade de Medicina de Ribeirão Preto da Universidade de São Paulo Avenida Bandeirantes, 3.900 - Campus Universitário - Monte Alegre CEP 14048-900 - Ribeirão Preto/SP Fone: (16) 3602-1000/ Fax: (16) 3633-1144
Trabalho realizado no Departamento de Ginecologia e Obstetrícia da Faculdade de Medicina de Ribeirão Preto da Universidade de São Paulo - USP; Hospital das Clínicas da Faculdade de Medicina de Ribeirão Preto da Universidade de São Paulo -USP - Ribeirão Preto (SP), Brasil.

Médicos Assistentes do Setor de Oncologia Ginecológica e Mastologia do Hospital das Clínicas da Faculdade de Medicina de Ribeirão Preto da Universidade de São Paulo - USP - Ribeirão Preto (SP), Brasil.

2 Professor Titular do Departamento de Ginecologia e Obstetrícia da Faculdade de Medicina de Ribeirão Preto da Universidade de São Paulo - USP - Ribeirão Preto (SP), Brasil; Coordenador do Setor de Oncologia Ginecológica e Mastologia do Hospital das Clínicas da Faculdade de Medicina de Ribeirão Preto da Universidade de São Paulo - HC/FMRP-USP - Ribeirão Preto (SP), Brasil.

3 Doutor, Professor do Departamento de Ginecologia e Obstetrícia da Faculdade de Medicina de Ribeirão Preto da Universidade de São Paulo - USP - Ribeirão Preto (SP), Brasil.

${ }_{4}^{4}$ Professor Associado do Departamento de Ginecologia e Obstetrícia da Faculdade de Medicina de Ribeirão Preto da Universidade de São Paulo - USP - Ribeirão Preto (SP), Brasil.

${ }_{5}^{5}$ Pós-graduando do Departamento de Ginecologia e Obstetrícia da Faculdade de Medicina de Ribeirão Preto da Universidade de São Paulo - USP - Ribeirão Preto (SP), Brasil. 
normal distribution (such as number of axillary nodes with metastasis and follow-up duration), the Kruskal-Wallis' test was used. For delineating the curves of free-of-disease and global survival, the log-rank test was used. RESULTS: there was no difference among the groups in the stage distribution, concerning the tumoral differentiation grade or in the distribution of histological types, and in the estrogen receptor and c-erb-B2 expression. Difference was found in the $\mathrm{RP}$ expression, which was less frequent in the group of patients under 40 , than in the group of patients over 50 (36.2\% versus $58.4 \%$ ) respectively. There was no difference among the groups in the mean tumoral diameter $(5.1,4.7$ and $5 \mathrm{~cm}$, respectively). There was also no difference among the groups, concerning the rate of axillary lymph node metastasis $(63.9,46.9$ and $50 \%$, respectively). The average follow-up was 54 months for all the groups. Disease recurrence occurred in $22.6 \%$ of patients under 40 years old, in $60 \%$ of patients between 40 and 50 , and in $22.6 \%$ of patients over 50 , with a significant difference among groups $(p<0.0001)$. Death caused by the disease was higher among patients under $40(46.9 \%)$ than among patients between 40 and $50(26.9 \%)$ and over $50(22.6 \%), p=0.0019$. The logistic analysis showed that "age under 40 " and the "presence of more than one metastatic axillary node" were independent death risk factors. CONCLUSIONS: age under 40 is an independent risk factor for breast cancer. The traditional prognostic indicators, such as stage, tumoral diameter, axillary involvement and presence of hormonal receptors are not associated with the disease evolution.

\section{Introdução}

A ocorrência de câncer de mama em mulheres jovens é um evento infreqüente. Cerca de $6,5 \%$ dos casos desta neoplasia ocorrem em mulheres com menos de 40 anos e $0,6 \%$, em mulheres com menos de 30 anos $^{1}$. A idade é, com freqüência, mencionada como um fator independente de mau prognóstico, embora esta conclusão não seja uniformemente aceita ${ }^{2}$. Em algumas séries de casos, vários fatores clínicos e epidemiológicos de mau prognóstico são associados às faixas etárias mais baixas ${ }^{3,4}$.

Em relação à história familiar, maior freqüência de casos de câncer de mama em parentes de primeiro grau é descrita em mulheres mais jovens. Em uma série de casos, a porcentagem de história familiar positiva chegou a $24 \%$ das pacientes ${ }^{3}$. Uma explicação para esta frequiência é o achado de mutações nos genes BRCA-1 e BRCA-2, presentes em até $12,8 \%$ dos casos no grupo mais jovem ${ }^{5}$. Nas faixas etárias mais elevadas, esta proporção foi de 5,2\% .

Por outro lado, com relação ao indicador de prognóstico mais relevante - o estádio -, as análises são conflitantes. Em uma série de casos, mulheres mais jovens apresentaram tumores com maiores diâmetros no momento do diagnóstico. Cerca de $29 \%$ das neoplasias foram diagnosticadas no estádio I, comparadas com $47 \%$ em pacientes com mais de 40 anos. Em $65 \%$ dos casos, os tumores eram estádios II e III em mulheres com menos de 40 anos de idade, e 47,9\% nas pacientes mais velhas ${ }^{4}$. Esta diferença não foi observada em outro estudo ${ }^{7}$. Taxas mais elevadas de acometimento de linfonodos axilares é descrita em pacientes com menos de 40 anos de idade quando comparadas com pacientes mais velhas ( 52,5 versus $41,8 \%$, respectivamente). Esta diferença é evidenciada tanto na avaliação clínica como na análise histológica ${ }^{7,8}$.

Algumas características histológicas dos tumores em mulheres mais jovens já foram analisadas. Entre as diferenças observadas, em relação às pacientes mais idosas, menciona-se o grau histológico mais alto. A proporção de casos com graduação histológica 3 pela escala de Bloom e Richardson varia entre 59 e $64 \%$, sendo esta característica associada a maior mortalidade pela doença ${ }^{8,9}$. São também relatadas freqüências significativamente mais elevadas de aneuploidia entre os casos com menos de 40 anos comparados às pacientes com idade mais elevada ( 78 contra $58 \%$ ) e, também, maior proporção de células neoplásicas com marcadores de proliferação celular (48 contra $26 \%)^{3}$.

A literatura ainda é controversa quanto às taxas de recorrência local e sistêmica entre as mulheres jovens. A porcentagem de recorrência loco-regional da doença varia de 12 a $35 \%$, valores significativamente mais elevados do que os relatados em pacientes de mais idade no diagnóstico. O risco de recidiva local da doença chega a ser cinco vezes mais elevado, quando se comparam as pacientes com menos de 35 anos e as com mais de $60 \operatorname{anos}^{10,11}$. No entanto, enquanto em algumas séries a idade se mostrou um fator independente de risco para recorrência local tanto após tratamento conservador da mama como após cirurgia radical ${ }^{10,12}$, em outras não se encontrou diferença na taxa de recidivas locais entre os casos tratados com cirurgia radical, mas apenas entre os casos para os quais se empregou cirurgia conservadora ${ }^{1}$.

A idade, na ocasião do diagnóstico, também é descrita como fator preditivo de maior risco. De acordo com uma revisão européia com mais de 1.700 casos, o risco relativo para desenvolvimento de metástases durante o seguimento foi mais de duas vezes maior em pacientes com menos de 40 anos, em comparação com pacientes mais velhas ${ }^{11}$. Em relação aos índices de sobrevida global, também não há consenso de que a idade isoladamente seja fator de risco para morte pela doença. Alguns estudos mostram que as curvas de sobrevida global são semelhantes nas diferentes faixas etárias pareadas por estádio, tanto para pacientes submetidas a tratamento conservador da mama quanto entre as submetidas a mastectomia ${ }^{13}$, enquanto outros demonstram impacto da idade sobre o risco para morte por câncer ${ }^{14,15}$.

Um estudo nacional descreveu as características epidemiológicas e clínicas de uma série de pacientes com idade inferior a $35 \operatorname{anos}^{16}$. No entanto, não há estudos controlados analisando as características epidemiológicas e clínicas que diferenciam as pacientes mais jovens com 
câncer de mama e sua associação com o prognóstico. $\mathrm{O}$ objetivo primário deste estudo foi comparar os indicadores de prognóstico em pacientes com menos de 40 anos com grupos de faixa etária mais elevada.

\section{Métodos}

Foi desenvolvido um estudo retrospectivo do tipo caso-controle com três grupos de análise. O grupo de Casos (menos de 40 anos no diagnóstico) e dois grupos controle em diferentes faixas etárias (Controles I, com pacientes entre 40 e 50 anos, e Controles II, com pacientes com mais de 50 anos). Todas as informações foram obtidas com a análise dos prontuários das pacientes. Foram incluídas no estudo pacientes atendidas seqüencialmente no Ambulatório de Mastologia do Hospital das Clínicas de Ribeirão Preto, com a primeira consulta entre janeiro de 1994 e dezembro de 2004. O protocolo da pesquisa e a dispensa do termo de consentimento livre e esclarecido foram aprovados pelo Comitê de Ética em Pesquisa da instituição.

Todas as pacientes apresentavam diagnóstico histológico de carcinoma invasor da mama. Os critérios de inclusão no estudo foram comuns aos três grupos: sexo feminino, sem antecedentes de outras neoplasias malignas (exceção o carcinoma de pele não melanoma), atendimento inicial entre janeiro de 1994 e dezembro de 2004 e sem tratamento prévio em outro serviço para a doença atual. Foram excluídas da análise as pacientes com doença metastática no diagnóstico (que foram nove casos no grupo de pacientes com menos de 40 anos e seis casos nos dois Grupos Controles).

No Grupo Casos, foram incluídas 75 pacientes com menos de 40 anos de idade na ocasião do diagnóstico. $\mathrm{O}$ Grupo Controles I foi formado por 68 pacientes com mais de 40 anos completos até 50 anos de idade e o Grupo Controles II incluiu 75 pacientes de 50 anos completos ou mais.

A inclusão das pacientes dos Grupos Controles foi determinada pela data da primeira consulta no Ambulatório de Mastologia. A partir da entrada no serviço de mastologia de uma paciente com menos de 40 anos com diagnóstico de câncer de mama, a próxima paciente com idade entre 40 e 50 anos e a próxima com mais de 50 anos incluídas na rotina de tratamento do serviço foram selecionadas para os Grupos Controles. A inclusão das pacientes pelo critério de atendimento consecutivo foi feita para se evitar viés de seleção. A mediana de idade no grupo de pacientes jovens foi de 35 anos (variando entre 22 e 39 anos). Nos Grupos Controles, as medianas foram respectivamente de 44 (40-49) e 58 (50-83) anos.

O estadiamento da doença incluiu exame clínico geral, mamário e das cadeias linfáticas loco-regionais (axila e fossa supraclavicular). No exame das mamas, foram anotadas as lesões clinicamente detectáveis, suas dimensões e demais alterações. Mamografias foram obtidas para todos os casos e, quando indicado, ultra-sonografia das mamas. Para lesões não palpáveis, as pacientes foram submetidas à biópsia por estereotaxia. Para rastreamento de metástases, foram empregadas a ultra-sonografia ou tomografia computadorizada de abdome, cintilografia óssea e radiografia ou tomografia computadorizada de tórax. As pacientes foram estadiadas seguindo as categorias propostas pela International Union Against Cancer (UICC) ${ }^{17}$.

Em todas as faixas etárias, a quimioterapia adjuvante foi oferecida para todas as pacientes com tumor maior que $1 \mathrm{~cm}$ no maior diâmetro patológico (pT1c) ou com metástase axilar diagnosticada em estudo histológico, índice de Karnofsky maior que 70\%. Pacientes com idade acima de 75 anos não foram submetidas à quimioterapia. O protocolo de quimioterapia seguiu o esquema CMF (ciclofosfamida $600 \mathrm{mg} / \mathrm{m}^{2}$, metotrexate $60 \mathrm{mg} / \mathrm{m}^{2}$ e 5 -fluoruracil $600 \mathrm{mg} / \mathrm{m}^{2}$ em infusão IV no D1 a cada 21/21 dias), para pacientes com três ou menos linfonodos axilares positivos, ou esquema FEC (5-fluoruracil $600 \mathrm{mg} / \mathrm{m}^{2}$, epirrubicina $60 \mathrm{mg} / \mathrm{m}^{2}$ e ciclofosfamida $600 \mathrm{mg} / \mathrm{m}^{2}$ em infusão IV no D1 a cada $21 / 21$ dias), para pacientes com mais de três linfonodos axilares positivos.

Hormonioterapia adjuvante com tamoxifeno $20 \mathrm{mg}$ vo foi oferecida para todas as pacientes com expressão positiva de receptores hormonais ou pacientes menopausadas (menopausa há mais de um ano ou ooforectomia bilateral) com receptores hormonais desconhecidos, por um período de 60 meses ou até recorrência da doença. A hormonioterapia adjuvante foi iniciada após a quimioterapia adjuvante ou após a cirurgia em pacientes que não receberam quimioterapia.

Radioterapia adjuvante foi administrada com técnica padrão utilizando campos medial e lateral tangenciais até atingir a dose total de $50 \mathrm{~Gy}$, com uma dose de $2 \mathrm{~Gy}$ por fração, em todo o tecido mamário residual. O tratamento foi diário, cinco dias por semana por seis semanas, após o término da quimioterapia ou após a recuperação da cirurgia em pacientes que não foram submetidas à quimioterapia adjuvante. A fossa supraclavicular foi irradiada em todas as pacientes com mais de três metástases em linfonodos axilares.

Os casos com doença localmente avançada (estádios clínicos IIB, IIIA e IIIB) receberam inicialmente tratamento neoadjuvante (quimio ou hormonioterapia), seguido de cirurgia conservadora ou mastectomia (dependendo do grau de resposta tumoral ao tratamento primário), além da linfadenectomia axilar unilateral. A classificação da resposta clínica ao tratamento neoadjuvante segue os padrões utilizados no estudo NSABP- $18^{18}$. Em resumo, considerou-se resposta completa quando houve desaparecimento da lesão alvo mensurável, e resposta parcial, a diminuição dos diâmetros do tumor em mais de $50 \%$. 
Se, após dois ciclos do tratamento primário, se constatasse aumento do produto dos diâmetros até $25 \%$, ou redução menor do que $50 \%$, classificava-se como doença estável; ocorrendo aumento do produto dos diâmetros em mais de $25 \%$, considerava-se doença progressiva. Nestas duas últimas situações, não havendo condições locais para cirurgia, indicava-se a radioterapia concomitante à quimioterapia.

Não houve diferença significante quanto ao número de pacientes submetidas ao tratamento neoadjuvante nos três grupos. Não foi evidenciada diferença nas taxas de resposta ao tratamento primário nos três grupos, sendo observadas porcentagens de respostas objetivas em 69,8, 78,6 e 75,7\% (à quimioterapia ou hormonioterapia) nos Grupos Casos, Controles I e Controles II. Esta semelhança entre as taxas de resposta teve impacto sobre as modalidades de tratamento cirúrgico empregado, pois não houve diferença entre as taxas de cirurgias conservadoras ou de cirurgia radical, com porcentagens de cirurgia conservadora de 61,4, 70,6 e 68\%, respectivamente, nas pacientes com menos de 40 anos, entre 40 e 50 anos e com mais de 50 anos $(\mathrm{p}=0,4)$.

O seguimento padrão após o tratamento foi baseado na reavaliação clínica (interrogatório dirigido e exame físico) com intervalos trimestrais no primeiro ano; semestral, no segundo e terceiro anos; e anual, a partir do quarto ano de tratamento. A mamografia foi solicitada seis meses após o tratamento primário e, a partir deste período, anualmente. Exames complementares somente foram realizados durante o seguimento caso houvesse indicação clínica. A média do tempo de seguimento foi de 54,1 meses (variando de 1 a 144, com mediana de 52) para pacientes jovens, 58,7 meses (variando de 1 a 166, com mediana de 50,5 meses) para as pacientes entre $40 \mathrm{e}$ 50 anos e 54,1 meses (variando de 2 a 99, com mediana de 52,5 meses) para as pacientes com mais de 50 anos. Não houve diferença para o tempo de seguimento entre os três grupos.

Foram coletados e analisados os dados sobre a idade no momento do diagnóstico, maior diâmetro das lesões, o produto dos dois maiores diâmetros perpendiculares, estadiamento clínico da doença, tipo histológico, exame histológico da peça cirúrgica e o estado dos linfonodos axilares. A presença de receptores hormonais nos tumores foi avaliada por imunohistoquímica, empregando-se anticorpos monoclonais (Novocastra), considerando-se positivos os tumores com mais de $10 \%$ de células com marcação. Somente foram empregadas as informações sobre as características histológicas dos tumores para os quais houvesse material disponível para análise.

As características do tratamento neoadjuvante (número de ciclos, intervalos entre os mesmos, esquema e dose, resposta ao tratamento primário) e a cirurgia a que a paciente foi submetida foram também coletadas. Foram calculados o intervalo livre de doença, a sobrevida global, a taxa de recidiva loco-regional ou a distância e a causa do óbito.

A distribuição das variáveis indicadas foi analisada e comparada entre os três grupos. O objetivo principal foi comparar os grupos quanto à sobrevida global, ao intervalo livre de doença e aos fatores associados aos desfechos. Para regressão logística, foram considerados: idade, diâmetro dos nódulos e presença ou não de gânglios axilares positivos para todos os grupos. Além disso, analisamos a freqüência de cirurgias radicais ou conservadoras e as taxas de recidiva e de óbito por câncer em cada grupo.

Utilizamos o teste do $X^{2}$ para as variáveis qualitativas (estádio clínico, grau de diferenciação tumoral, tipo histológico, receptores hormonais e c-erb-B2, uso de tratamento neoadjuvante e resposta ao mesmo, prescrição de hormonioterapia, emprego de radioterapia, tipo de cirurgia e grau de acometimento axilar, recidivas da doença e óbitos). Para as variáveis quantitativas que não apresentam distribuição normal (como número de gânglios axilares acometidos por metástases e tempo de seguimento) foi utilizado o teste de Kruskal-Wallis. Para a construção das curvas de sobrevida livre de doença e sobrevida global, foi utilizado o teste log-rank.

\section{Resultados}

O tipo histológico predominante nos três grupos foi o ductal invasor, com 85,5, 89,3 e 81,8\% dos casos nos Grupos Casos, Controles I e Controles II, respectivamente $(\mathrm{p}=0,4)$. A distribuição por estádio clínico foi semelhante nos três grupos $(\mathrm{p}=0,14)$. No Grupo Casos, $38,6 \%$ das pacientes foram diagnosticadas no estádio I ou IIA (27 pacientes) e $61,4 \%$ das pacientes foram classificadas como tumores localmente avançados (estádios IIB, IIIA e IIIB, 43 pacientes). No Grupo Controles I, a proporção de tumores iniciais e localmente avançados foi de 47,1 e $52,9 \%$, respectivamente (32 e 36 pacientes). No Grupo Controles II, a proporção de tumores iniciais e localmente avançados foi de 41,3 e 58,7\%, respectivamente ( 31 e 44 pacientes, $\mathrm{p}=0,4$ ).

Não houve diferença significante quanto ao maior diâmetro tumoral no diagnóstico $(\mathrm{p}=0,8)$. As médias foram respectivamente de $5,1 \mathrm{~cm}$ (variando entre 0,6 e $15 \mathrm{~cm}$ ) para o Grupo Casos, 4,7 cm no Grupo Controles I (entre 1 e $19 \mathrm{~cm}$ ) e de $5 \mathrm{~cm}$ no grupo de pacientes com mais de 50 anos de idade (variando entre 1 e $14 \mathrm{~cm}$ ). Embora a média do diâmetro tumoral no Grupo Casos tenha sido maior, também não houve diferença na proporção de tumores $\mathrm{T} 1, \mathrm{~T} 2, \mathrm{~T} 3$ ou $\mathrm{T} 4(\mathrm{p}=0,61)$.

Quanto à presença de receptores hormonais, houve diferença significante entre os grupos somente 
em relação aos receptores de progesterona, que foram menos freqüentemente encontrados nos tumores de mulheres com menos de 40 anos, em relação às pacientes com mais de 50 anos $(p=0,006)$. A porcentagem de tumores positivos para receptores de progesterona foi de 36,2, 52,8 e 60,3\% para os Grupos Casos, Controles I e Controles II, respectivamente. Não houve diferença significante quanto à expressão de receptores de estrógenos entre os grupos $(58,7,64,9$ e 72,3\%, respectivamente). Quanto à expressão da proteína c-erb-B2 não houve diferenças entre os três grupos, com taxas de positividade de 30,5, 22,6 e $23,1 \%$, respectivamente nos Grupos Casos, Controles I e Controles II. Também não observamos diferença na distribuição por graus de diferenciação tumoral $(p=0,3)$. As taxas de tumores indiferenciados ( $\mathrm{grau}$ III) foram de 22,2, 23 e 11,9\%, respectivamente, nos Grupos Casos, Controles I e Controles II.

A mediana do número de gânglios axilares comprometidos por neoplasia foi de 1,0 no Grupo Casos, zero no Grupo Controles I e 1,0 no Grupo Controles II. O número de mulheres com gânglios positivos foi de $40(57,1 \%)$ no Grupo Casos, $31(45,6 \%)$ no Grupo Controles I e 37 (49,3\%) no Grupo Controles II, sem diferença significante entre os grupos (Tabela 1).

Durante o período de seguimento, no Grupo Casos, houve 42 casos de recorrência da doença. Nos Grupos Controles, o número de recidivas foram respectivamente de 22 e 17 nas pacientes do Grupo Controles I e com mais de 50 anos. Houve diferença significante nas freqüências de recidivas entre o Grupo Casos em relação aos dois Grupos Controles $(p<0,0001)$, mas não entre os dois Grupos Controles $(\mathrm{p}=0,2)$.

Quando se verifica a mortalidade por câncer, nota-se que houve 31 óbitos ( $46,9 \%$ das pacientes) pela doença em pacientes com menos de 40 anos durante o tempo de seguimento. No grupo de pacientes entre 40 e 50 anos, houve 18 óbitos (26,9\% das pacientes) e no grupo das pacientes com mais de 50 anos ocorreram 11 óbitos (14,9\% das pacientes). Houve uma paciente do Grupo Casos que não se submeteu ao tratamento e teve um mês de sobrevida, não tendo sido feito o rastreamento completo para metástases (estádio clínico IIIA). Também houve uma paciente do Grupo Controles I com óbito por complicações do tratamento (neutropenia com morte por infecção pulmonar).

Observamos diferença significante quando comparamos o número de óbitos entre pacientes com menos de 40 anos com as pacientes de mais de 50 anos ( $\mathrm{p}=0,001)$, mas não houve diferença quando comparamos o Grupo Casos e Controles I ou Grupos Controles I e Controles II (Figura 1). As curvas de sobrevida mostraram diferença significante nas taxas de sobrevida quando se compara pacientes com menos de 40 anos de idade tanto em relação às pacientes do Grupo Controles II ( $\mathrm{p}=0,0002)$ quanto às do Grupo Controles I $(p=0,03)$. Não houve diferenças nas taxas de sobrevida entre as pacientes do Grupo Controles I e as do Grupo Controles II $(p=0,08)$, conforme Tabela 2.

Tabela 1 - Características histológicas dos tumores das pacientes nos três grupos, tipo de cirurgia e presença de gânglios axilares metastáticos nos três grupos

\begin{tabular}{|c|c|c|c|c|c|c|}
\hline \multirow{3}{*}{$\begin{array}{l}\text { Classificação Bloon } \\
\text { e Richardson }\end{array}$} & \multicolumn{2}{|c|}{ Casos } & \multicolumn{2}{|c|}{ Controles I } & \multicolumn{2}{|c|}{ Controles II } \\
\hline & n & $\%$ & n & \& & n & $\%$ \\
\hline & & & & & & \\
\hline Total & \multicolumn{2}{|c|}{63} & \multicolumn{2}{|c|}{61} & \multicolumn{2}{|c|}{59} \\
\hline Grau I & 14 & 22,2 & 18 & 29,5 & 19 & 32,2 \\
\hline Grau II & 35 & 55,6 & 29 & 47,5 & 33 & 55,9 \\
\hline Grau III & 14 & 22,2 & 14 & 23,0 & 7 & 11,9 \\
\hline \multicolumn{7}{|l|}{$\begin{array}{l}\text { Receptores } \\
\text { de estrógenos }\end{array}$} \\
\hline Total & \multicolumn{2}{|c|}{62} & \multicolumn{2}{|c|}{57} & \multicolumn{2}{|c|}{68} \\
\hline Positivo & 36 & 58,1 & 36 & 63,1 & 49 & 72,1 \\
\hline Negativo & 26 & 41,9 & 21 & 36,9 & 19 & 27,9 \\
\hline \multicolumn{7}{|l|}{$\begin{array}{l}\text { Receptores } \\
\text { de progesterona }\end{array}$} \\
\hline Total & \multicolumn{2}{|c|}{58} & \multicolumn{2}{|c|}{53} & \multicolumn{2}{|c|}{65} \\
\hline Positivo & 21 & 36,2 & 28 & 52,8 & 38 & 58,4 \\
\hline Negativo & 37 & 63,8 & 25 & 47,2 & 27 & 41,6 \\
\hline \multicolumn{7}{|c|}{ Modalidade cirúrgica } \\
\hline Total & \multicolumn{2}{|c|}{70} & \multicolumn{2}{|c|}{68} & \multicolumn{2}{|c|}{71} \\
\hline Conservadora & 43 & 61,4 & 48 & 67,7 & 50 & 70,4 \\
\hline Radical & 27 & 38,6 & 20 & 32,3 & 21 & 29,6 \\
\hline \multicolumn{7}{|l|}{$\begin{array}{l}\text { Gânglios } \\
\text { comprometidos }\end{array}$} \\
\hline Total & \multicolumn{2}{|c|}{72} & \multicolumn{2}{|c|}{66} & \multicolumn{2}{|c|}{72} \\
\hline Zero & 31 & 43,1 & 35 & 53,1 & 36 & 50,0 \\
\hline Até 3 & 21 & 29,2 & 16 & 24,2 & 12 & 16,7 \\
\hline 4 ou mais & 20 & 27,7 & 15 & 22,7 & 24 & 33,3 \\
\hline
\end{tabular}

Casos: pacientes com menos de 40 anos; Controles I: pacientes com idade entre 40 e 50 anos; Controles II: pacientes com mais de 50 anos de idade.

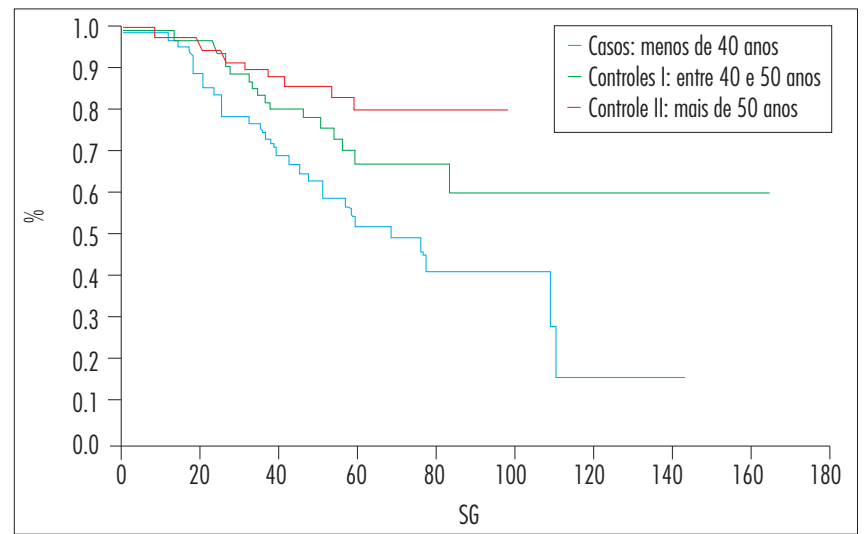

Figura 1 - Sobrevida global (SG) em pacientes com câncer de mama em três diferentes grupos divididos por faixa etária. Casos: com idade inferior a 40 anos; Controles I: com idade acima de 40 anos e abaixo de 50 anos; e Controles II: com idade acima de 50 anos 
Tabela 2 - Número absoluto e porcentagem de recidivas e óbitos pela doença durante 0 período de seguimento nos três grupos

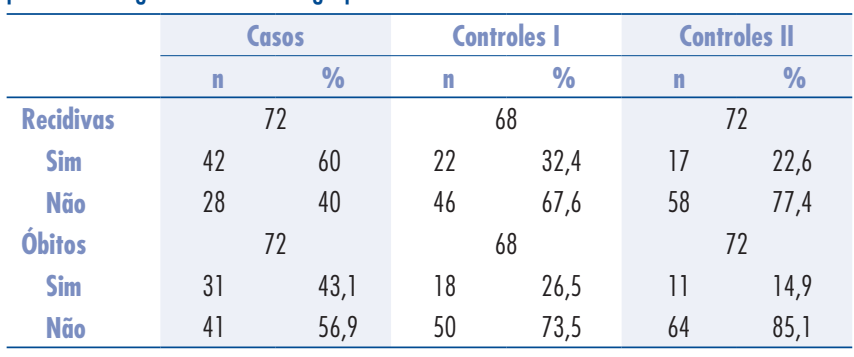

Casos: pacientes com menos de 40 anos; Controles I: pacientes com idade entre 40 e 50 anos; Controles II: pacientes com mais de 50 anos de idade.

De todas as variáveis analisadas, a idade, estádio clínico, diâmetro clínico do tumor maior do que $2 \mathrm{~cm}$, presença de gânglios axilares palpáveis, emprego de quimioterapia neoadjuvante (que reflete a porcentagem de casos com tumores localmente avançados) e de cirurgia conservadora tiveram correlação significante com a morte por câncer. Com a regressão logística, evidenciou-se que a idade de menos de 40 anos no diagnóstico e a presença de mais de um gânglio axilar acometido por metástase foram fatores independentes relacionados à morte por câncer.

\section{Discussão}

Os aspectos clínico-patológicos que poderiam justificar o fato de o câncer de mama em mulheres jovens ser conhecido como de pior prognóstico foram analisados. A casuística coletada é representada por características especiais que refletem o atendimento em um centro de referência com alta porcentagem de casos com doença localmente avançada. Por outro lado, estas características permitiram comparar mais adequadamente os desfechos. A inclusão de dois Grupos Controle - um dos quais composto por pacientes com idade entre 40 e 50 anos - permitiu comparação com mulheres mais próximas biologicamente do grupo de estudo.

Embora seja conhecida a tendência das neoplasias de se apresentarem em estádios clínicos mais avançados entre as pacientes mais jovens ${ }^{2,4,19,20}$, observamos que a distribuição por estádios foi semelhante em todos os grupos do estudo. Esta semelhança entre os estádios nos três grupos pode ser antecipada quando se comparam as médias dos diâmetros tumorais, que foram de $5,1 \mathrm{~cm}$ no grupo de pacientes jovens, 4,7 para a faixa entre 40 e 50 anos e $5 \mathrm{~cm}$ entre as que tinham mais de 50 anos. Dados similares foram descritos por outros autores ${ }^{7,21}$. Na maior parte das séries, no entanto, as mulheres jovens com câncer de mama apresentam tumores de maior diâmetro e estádios mais avançados ${ }^{2,4}$. Nossa casuística apresenta características diferentes da literatura quanto aos diâmetros dos tumores e estadiamento, com maior freqüência de casos em estádios mais avançados, em todos os grupos de idade.
Em associação com os resultados referentes ao estádio, notamos também semelhanças quanto a vários outros indicadores de prognóstico habitualmente empregados para análise. Assim, as pacientes com mais de 40 anos apresentaram proporções semelhantes de expressão de receptores hormonais em relação às pacientes jovens. O único indicador de prognóstico com distribuição diferente nos três grupos foi a expressão de receptores de progesterona, com menor porcentagem de tumores expressando este receptor no grupo de pacientes com menos de 40 anos e porcentagens semelhantes entre si nos dois outros grupos. Resultado semelhante já foi descrito em estudo com pacientes muito jovens (menos de 35 anos), com taxas de positividade de 36,7 e $43,5 \%$ no grupo controle $^{21}$. Por outro lado, é incerto o papel dos receptores de progesterona como indicador de prognóstico, havendo evidências favoráveis ${ }^{22,23}$ e contrárias ${ }^{24}$. Também não encontramos diferenças significativas em relação ao grau de diferenciação tumoral entre os três grupos. $\mathrm{O}$ grau de acometimento axilar por metástases (mais de um gânglio acometido) também foi um fator independente de risco de morte pela doença, porém não variou significativamente entre os diferentes grupos etários. Esta semelhança, quanto à freqüência dos indicadores clássicos de sobrevida entre grupos de diferentes idades, é encontrada em algumas séries $^{2,21}$ mas não em outras ${ }^{3,9,19,20}$.

A distribuição da freqüência de indicadores de prognóstico entre as jovens mostra que, na maior parte dos estudos, as diferenças são devidas à seleção de casos e critérios de inclusão $0^{4,21}$. Além disso, as comparações são dificultadas pelas diferentes faixas etárias incluídas e pela variação nos parâmetros analisados ${ }^{3}$. Em estudos envolvendo grandes amostras de base populacional, observa-se que as mulheres mais jovens com carcinoma de mama tendem a apresentar estádios mais avançados e achados correlacionados, como tumores menos diferenciados e menor porcentagem de tumores com receptores hormonais ${ }^{4}$.

Com relação ao tratamento, não houve diferença nas taxas de indicação e prescrição de tratamento neoadjuvante. Da mesma forma, não encontramos diferença nas taxas de resposta a esta modalidade de tratamento e também quanto às indicações de cirurgia radical ou conservadora, com porcentagens semelhantes ao relatados em estudos sobre o tema ${ }^{7,13,19}$. No entanto, apesar da semelhança quanto aos fatores de prognóstico de uso corrente e nas formas de tratamento, as pacientes mais jovens apresentam recidivas mais freqüentes e taxas de sobrevida livre e global inferiores às pacientes mais velhas ${ }^{21}$ - e esta diferença permanece mesmo quando se estratifica as pacientes por estádio ${ }^{4}$. Na nossa casuística, a freqüência de óbitos pela doença é quase três vezes maior no Grupo Casos do que nas pacientes com mais de 50 anos. Esta diferença foi encontrada já nas fases iniciais do seguimento e, nas 
avaliações mais tardias, aos cinco e dez anos. A letalidade da doença continua mais elevada entre as pacientes jovens, com uma sobrevida global em torno de $60 \%$ em cinco anos neste grupo, contra $85 \%$ nas pacientes com mais de 50 anos. Várias explicações para esta diferença têm sido propostas. Uma delas explora a hipótese de que as mulheres jovens, cujos tumores expressem receptores para estrogênios e progesterona, não recebem tratamento adjuvante adequado ${ }^{25}$. Em nosso estudo, a proporção de casos que seriam candidatos a tratamento hormonal adjuvante é muito reduzida para que esta explicação seja suficiente para explicar a diferença.

As limitações do nosso estudo são devidas à inclusão de grande número de pacientes com tumores avançados em todas as faixas de idade e à extensão da casuística. No entanto, observa-se que a neoplasia de mama em pacientes jovens tem evolução claramente mais desfavorável, com letalidade significativamente mais elevada, mesmo que fatores clínicos e histológicos como estádio, diâmetro do tumor, acometimento axilar por metástases, grau histológico e receptores hormonais sejam semelhantes aos dos Grupos Controle.

Embora as características dos tumores possam ser responsabilizadas por parte da diferença, a análise multivariada, controlando-se pelos fatores mais relevantes (estádio, grau tumoral, uso de tratamento sistêmico etc.), estreita as diferenças entre os grupos de idades diferentes, mas as pacientes mais jovens permanecem com prognóstico pior ${ }^{4}$. Esta dissociação entre a distribuição semelhante dos indicadores de prognóstico e as diferenças nas taxas de sobrevida contribui para a hipótese de que a idade seja realmente um fator independente de mau prognóstico ${ }^{3}$. Outros autores, revisando o tema, chegaram a resultado semelhante ${ }^{26} \mathrm{e}$ concluem que os fatores de prognóstico convencionais não permitem diferenciar características especiais destes casos. Provavelmente, apenas o emprego de novas ferramentas como perfil de expressão gênico possa predizer com maior acurácia o prognóstico, tendo em vista que a proporção de pacientes com assinatura gênica de mau prognóstico é mais elevada entre as pacientes jovens ${ }^{26}$.

\section{Referências}

1. Arriagada R, Lê MG, Contesso G, Guinebretière JM, Rochard F, Spielmann M. Predictive factors for local recurrence in 2006 patients with surgically resected small breast cancer. Ann Oncol. 2002; 13(9): 1404-13.

2. Jmor S, Al-Sayer H, Heys SD, Payne S, Miller I, Ah-See A, et al. Breast cancer in women aged 35 and under: prognosis and survival. J R Coll Surg Edinb. 2002;47(5):693-9.

3. Sidoni A, Cavaliere A, Bellezza G, Scheibel M, Bucciarelli E. Breast cancer in young women: clinicopathological features and biological specificity. Breast. 2003;12(4):247-50.

4. Maggard MA, O'Connell JB, Lane KE, Liu JH, Etzioni DA, Ko CY. Do young breast cancer patients have worse outcomes? J Surg Res. 2003;113(1):109-13.

5. Loman $N$, Johannsson $O$, Kristoffersson $U$, Olsson $H$, Borg A. Family history of breast and ovarian cancers and BRCA 1 and BRCA2 mutations in a population-based series of early-onset breast cancer. J Natl Cancer Inst. 2001 ;93(16):1215-23.

6. Bonadona V, Sinilnikova OM, Chopin S, Antoniou AC, Mignotte $\mathrm{H}$, Mathevet $\mathrm{P}$, et al. Contribution of BRCA1 and BRCA2 germline mutations to the incidence of breast cancer in young women: results from a prospective population-based study in France. Genes Chromosomes Cancer. 2005;43(4):404-13.

7. Foo CS, Su D, Chong CK, Chng HC, Tay KH, Low SC, et al. Breast cancer in young Asian women: study on survival. ANZ J Surg. 2005;75(7):566-72.

8. Sundquist $M$, Thorstenson S, Brudin L, Wingren S, Nordenskjöld $B$. Incidence and prognosis in early onset breast cancer. Breast. 2002;11(1):30-5.

9. Fernandopulle SM, Cher-Siangang P, Tan PH. Breast carcinoma in women 35 years and younger: a pathological study. Pathology. 2006;38(3):219-22.
10. Zhou P, Gautam S, Recht A. Factors affecting outcome for young women with early stage invasive breast cancer treated with breast-conserving therapy. Breast Cancer Res Treat. 2007; 101(1):51-7.

11. Voogd AC, Nielsen M, Peterse JL, Blichert-Toft $M$, Bartelink $H$, Overgaard $M$, et al. Differences in risk factors for local and distant recurrence after breast-conserving therapy or mastectomy for stage I and II breast cancer: pooled results of two large European randomized trials. J Clin Oncol. 2001;19(6):1688-97.

12. Bollet MA, Sigal-Zafrani B, Mazeau V, Savignoni A, de la Rochefordière A, Vincent-Salomon A, et al. Age remains the first prognostic factor for loco-regional breast cancer recurrence in young ( $<40$ years) women treated with breast conserving surgery first. Radiother Oncol. 2007;82(3):272-80.

13. Kroman N, Holtveg $H$, Wohlfahrt J, Jensen MB, Mouridsen HT, Blichert-Toft $M$, et al. Effect of breast-conserving therapy versus radical mastectomy on prognosis for young women with breast carcinoma. Cancer. 2004;100(4):688-93.

14. Fowble BL, Schultz DJ, Overmoyer B, Solin LJ, Fox K, Jardines L, et al. The influence of young age on outcome in early stage breast cancer. Int J Radiat Oncol Biol Phys. 1994;30(1):23-33.

15. Broët $P$, de la Rochefordière $A$, Scholl SM, Fourquet $A$, De Rycke $Y$, Pouillart $P$, et al. Analyzing prognostic factors in breast cancer using a multistate model. Breast Cancer Res Treat. 1999;54(1):83-9.

16. Crippa CG, Hallal ALC, Dellagiustina AR, Traebert EE, Gondin G, Pereira C. Perfil clínico e epidemiológico do câncer de mama em mulheres jovens. ACM Arq Catarin Med. 2003;32(3):50-8.

17. Sobin $L H$, Wittekind $C$, editors. TNM classification of malignant tumours (UICC). 6th ed. New York: Wiley; 2002.

18. Fisher B, Bryant J, Wolmark N, Mamounas E, Brown A, Fisher ER, et al. Effect of preoperative chemotherapy on the outcome of women with operable breast cancer. J Clin Oncol. 1998;16(8):2672-85. 
19. Aebi S, Gelber S, Castiglione-Gertsch M, Gelber RD, Collins J, Thürlimann $B$, et al. Is chemotherapy alone adequate for young women with oestrogen-receptor-positive breast cancer? Lancet. 2000;355(9218):1869-74.

20. Gajdos C, Tartter PI, Bleiweiss IJ, Bodian C, Brower ST. Stage 0 to stage III breast cancer in young women. J Am Coll Surg. 2000; 190(5):523-9.

21. Han W, Kim SW, Park IA, Kang D, Kim SW, Youn YK, et al. Young age: an independent risk factor for disease-free survival in women with operable breast cancer. BMC Cancer. 2004;4:82.

22. Fisher B, Redmond C, Fisher ER, Caplan R. Relative worth of estrogen or progesterone receptor and pathologic characteristics of differentiation as indicators of prognosis in node negative breast cancer patients: findings from National Surgical Adjuvant Breast and Bowel Project Protocol B-06. J Clin Oncol. 1988;6(7):1076-87.
23. Yu KD, Liu GY, Di GH, Wu J, Lu JS, Shen KW, et al. Progesterone receptor status provides predictive value for adjuvant endocrine therapy in older estrogen receptor-positive breast cancer patients. Breast. 2007; 16(3):307-15.

24. Stierer M, Rosen H, Weber R, Hanak H, Auerbach L, Spona J, et al. A prospective analysis of immunohistochemically determined hormone receptors and nuclear features as predictors of early recurrence in primary breast cancer. Breast Cancer Res Treat. 1995;36(1):11-21.

25. Colleoni M, Rotmensz N, Peruzzotti G, Maisonneuve P, Orlando L, Ghisini $R$, et al. Role of endocrine responsiveness and adjuvant therapy in very young women (below 35 years) with operable breast cancer and node negative disease. Ann Oncol. 2006;17(10):1497-503.

26. Aebi S, Castiglione M. The enigma of young age. Ann Oncol. 2006; 17(10):1475-7. 